\title{
Estimation of Non-negative ODFs Using the Eigenvalue Distribution of Spherical Functions
}

\author{
Evan Schwab, Bijan Afsari, and René Vidal \\ Center for Imaging Science, Johns Hopkins University
}

\begin{abstract}
Current methods in high angular resolution diffusion imaging (HARDI) estimate the probability density function of water diffusion as a continuous-valued orientation distribution function (ODF) on the sphere. However, such methods could produce an ODF with negative values, because they enforce non-negativity only at finitely many directions. In this paper, we propose to enforce non-negativity on the continuous domain by enforcing the positive semi-definiteness of Toeplitzlike matrices constructed from the spherical harmonic representation of the ODF. We study the distribution of the eigenvalues of these matrices and use it to derive an iterative semi-definite program that enforces non-negativity on the continuous domain. We illustrate the performance of our method and compare it to the state-of-the-art with experiments on synthetic and real data.
\end{abstract}

Keywords: diffusion imaging, orientation distribution functions, spherical harmonics, Toeplitz matrices, eigenvalue distribution theorem.

\section{Introduction}

Diffusion magnetic resonance imaging (dMRI) uses the properties of water diffusion in biological tissues to reconstruct the 3-dimensional architecture of anatomical structures. Recent advances in this field, such as high angular resolution diffusion imaging (HARDI), have been able to compute the anisotropy of water molecules in the brain by measuring diffusion along multiple directions. This allows one to characterize the diffusion properties of biological tissues in terms of a probability density function on the sphere, otherwise known as the orientation distribution function (ODF).

In theory, an ODF must obey the axioms of a probability distribution, which include being non-negative and integrating to 1 over the sphere. However, existing ODF estimation methods based on a spherical harmonic $(\mathrm{SH})$ representation of the ODF [1 9] do not enforce the non-negativity constraint. As a consequence, due to noise and low order $\mathrm{SH}$ representation, the estimated ODFs may contain negative values. This is a problem in population studies, where one is interested in applying statistical methods to ODFs to differentiate between healthy and diseased populations, which cannot be accurately done without axiomatically correct distributions. To address this problem, [10] enforces non-negativity at finitely many directions on the sphere, but ODF interpolation and registration methods may require evaluating ODFs outside discrete grids. [1] uses a non-ODF constrained 
spherical deconvolution method that reduces the occurrence of negative values, but does not completely eliminate them.

In this paper, we propose an algorithm that enforces non-negativity for all directions on the sphere. To do this, we extend the relationship between continuous functions and their companion Toeplitz forms of Fourier coefficients to spherical functions. This leads to a positive semi-definiteness constraint on matrices constructed from the SH coefficients of the ODF. Since these constraints cannot be directly enforced, we study the distribution of the eigenvalues of these matrices to predict their smallest eigenvalue given an estimate of the SH coefficients. This prediction can in turn be used to improve the $\mathrm{SH}$ coefficients. This leads to an iterative semi-definite program (SDP) that enforces non-negativity of the ODF on the continuous domain. We illustrate the performance of our method in comparison to the state-of-the-art with experiments on synthetic and real data.

\section{Estimating ODFs Using Spherical Harmonics}

\subsection{Standard and Modified SH Basis Representations}

Recent ODF estimation methods have adopted the SH representation for HARDI signals. The (standard) SH basis elements are complex-valued functions defined as

$$
Y_{l}^{m}(\theta, \phi)=\sqrt{\frac{2 l+1}{4 \pi} \frac{(l-m) !}{(l+m) !}} P_{l}^{m}(\cos \theta) e^{i m \phi}, \quad l=0,1,2, \ldots, \quad-l \leq m \leq l,
$$

where $P_{l}^{m}$ is the associated Legendre polynomial of degree $l$ and order $m, \theta \in$ $[0, \pi]$, and $\phi \in[0,2 \pi)$. In practice, the signals we want to reconstruct are real. Hence, it is more convenient to use the modified $\mathrm{SH}$ basis functions, which are defined as

$$
Y_{j}= \begin{cases}\sqrt{2} \operatorname{Re}\left(Y_{l}^{|m|}\right) & \text { if }-l \leq m<0, \\ Y_{l}^{0} & \text { if } m=0, \\ \sqrt{2}(-1)^{m+1} \operatorname{Im}\left(Y_{l}^{m}\right) & \text { if } 0<m \leq l,\end{cases}
$$

where $\operatorname{Re}(\cdot)$ and $\operatorname{Im}(\cdot)$ are the real and imaginary parts, respectively, and $j \doteq$ $j(l, m)=\frac{l^{2}+l+2}{2}+m$ for $l=0,2,4, \ldots$ and $-l \leq m \leq l$. Notice that, for degree up to $L$, there are $R=\frac{(L+1)(L+2)}{2}$ basis elements. Often it suffices to consider the modified $\mathrm{SH}$ basis of degree up to $L=4$ correlating to $R=15$. Notice also that $\int_{\mathbb{S}^{2}} Y_{1}(\theta, \phi)=\int_{\mathbb{S}^{2}} \frac{1}{2 \sqrt{\pi}}=2 \sqrt{\pi}$ and $\int_{\mathbb{S}^{2}} Y_{j}(\theta, \phi)=0$ for $j>1$. Consider a real continuous function $f: \mathbb{S}^{2} \rightarrow \mathbb{R}$. Then we can write it as $f=\sum_{j=1}^{\infty} c_{j} Y_{j}$ using the modified basis in (2), where $\boldsymbol{c}=\left[c_{j}\right]$ are the real SH coefficients that parameterize $f$. This is equivalent to writing $f=\sum_{l, m}^{\infty} c_{l, m} Y_{l}^{m}$ using the standard SH basis in (1), where we define $\overline{\boldsymbol{c}}=\left[c_{l, m}\right]$ as the vector of standard SH coefficients. Given a real vector $\boldsymbol{c}$, we can obtain $\overline{\boldsymbol{c}}$ by the inverse mapping of $j(l, m)=\frac{l^{2}+l+2}{2}+m$, where $l=0,2,4, \ldots,-l \leq m \leq l$ with $c_{l, m}=0$ for $l>0$ odd. This gives the degree $l_{j}$ and order $m_{j}$ of $Y_{j}$ and defines a one-to-one mapping between the vectors $\boldsymbol{c}$ and $\overline{\boldsymbol{c}}$. For example, $\bar{c}_{0,0}=c_{1}, \bar{c}_{2,-2}=c_{2}, \bar{c}_{2,-1}=c_{3}$ and so forth. 


\subsection{ODF Estimation Problem and Prior Work}

Let $S_{0}$ be the baseline MRI signal and let $S(\theta, \phi)$ be the HARDI signal along $(\theta, \phi)$. Following [7], we define the ODF as $p(\vartheta, \varphi)=\frac{1}{4 \pi}+\frac{1}{16 \pi^{2}} F R T\left\{\nabla_{b}^{2} \ln (-\ln \right.$ $\left.\left.\left(\frac{S(\theta, \phi)}{S_{0}}\right)\right)\right\}$, where $F R T$ is the Funk-Radon transform, $\nabla_{b}^{2}$ is the Laplace-Beltrami operator on $\mathbb{S}^{2}, \vartheta \in[0, \pi]$ and $\varphi \in[0,2 \pi)$. Let $s(\theta, \phi) \doteq \ln \left(-\ln \left(\frac{S(\theta, \phi)}{S_{0}}\right)\right)=$ $\sum_{j=1}^{\infty} c_{j} Y_{j}(\theta, \phi)$. Since $\nabla_{b}^{2}\left(Y_{j}(\theta, \phi)\right)=-l_{j}\left(l_{j}+1\right) Y_{j}(\theta, \phi)$, and $F R T\left(Y_{j}(\theta, \phi)\right)=$ $2 \pi P_{l_{j}}(0) Y_{j}(\vartheta, \varphi)$, where $P_{l_{j}}(0)$ is the Legendre polynomial of degree $l_{j}$ at 0 , we have

$$
p(\vartheta, \varphi)=\frac{1}{4 \pi}+\frac{1}{16 \pi^{2}} \sum_{j=1}^{\infty}\left(-2 \pi P_{l_{j}}(0)\right) l_{j}\left(l_{j}+1\right) c_{j} Y_{j}(\vartheta, \varphi)=\sum_{j=1}^{\infty} \tilde{c}_{j} Y_{j}(\vartheta, \varphi),
$$

where $\tilde{c}_{1}=\frac{1}{2 \sqrt{\pi}}$ and $\tilde{c}_{j}=-\frac{1}{8 \pi} P_{l_{j}}(0) l_{j}\left(l_{j}+1\right) c_{j}$ for $j>1$. Therefore, to reconstruct $p$, it suffices to compute the $\mathrm{SH}$ coefficients $\tilde{\boldsymbol{c}} \doteq\left[\tilde{c}_{j}\right]$ that parameterize the signal $s$, or equivalently, the standard SH coefficients $\overline{\tilde{\boldsymbol{c}}} \doteq\left[\tilde{c}_{l, m}\right]$, such that $\int_{\mathbb{S}^{2}} p=1$ and $p(\vartheta, \varphi) \geq 0$ for all $\vartheta \in[0, \pi]$ and $\varphi \in[0,2 \pi)$. To that end, assume that the HARDI signals are measured at $G$ gradient directions $\left(\theta_{i}, \phi_{i}\right)_{i=1}^{G}$. If we use an $R$-dimensional approximation of $s \approx \sum_{j=1}^{R} c_{j} Y_{j}$, we have $s \approx \mathbf{B} \boldsymbol{c}$, where $\boldsymbol{s} \doteq\left[\ln \left(-\ln \left(\frac{S\left(\theta_{1}, \phi_{1}\right)}{S_{0}}\right)\right), \ldots, \ln \left(-\ln \left(\frac{S\left(\theta_{G}, \phi_{G}\right)}{S_{0}}\right)\right)\right]^{T}, \mathbf{B}$ is the $G \times R$ SH basis matrix whose $i$-th row is $\mathbf{B}_{i}=\left[Y_{1}\left(\theta_{i}, \phi_{i}\right), \ldots, Y_{R}\left(\theta_{i}, \phi_{i}\right)\right]$, and $\boldsymbol{c}=\left[c_{1}, c_{2}, \ldots, c_{R}\right]^{T} \in \mathbb{R}^{R}$. With the above notation, we define the following ODF estimation problem:

Problem 1 (Continuous Domain ODF Estimation). Fix $L$ and let $\boldsymbol{c} \in \mathbb{R}^{R}$. Solve $\min _{\boldsymbol{c}}\|\mathbf{B} \boldsymbol{c}-\boldsymbol{s}\|_{2}^{2}$ s.t. $p_{L}(\vartheta, \varphi) \doteq \sum_{j=1}^{R} \tilde{c}_{j} Y_{j}(\vartheta, \varphi) \geq 0$ for all $\vartheta \in[0, \pi], \varphi \in[0,2 \pi)$.

Perhaps the simplest approach to recovering $\boldsymbol{c}$ is to solve the least-squares problem $\min _{\boldsymbol{c}} \frac{1}{2}\|\mathbf{B} \boldsymbol{c}-\boldsymbol{s}\|_{2}^{2}$, as proposed in [5]. However, disregarding the nonnegativity constraints could result in negative values for $p(\varphi, \vartheta)$. To address this issue, [10] proposes to enforce the non-negativity constraints at finitely many directions $\left(\vartheta_{i}, \varphi_{i}\right)_{i=1}^{M}$, with $\left(\vartheta_{i}, \varphi_{i}\right)$ possibly different from $\left(\theta_{i}, \phi_{i}\right)$ 1 To solve this problem, [10] defines the discrete ODF $\boldsymbol{p} \in \mathbb{R}^{M}$ whose $i$-th entry is $p_{i}=$ $p_{L}\left(\vartheta_{i}, \varphi_{i}\right)$. Then $\boldsymbol{p}=\frac{1}{4 \pi} \mathbf{1}+\frac{1}{16 \pi^{2}} \mathbf{C L P} \boldsymbol{c}$, where $\mathbf{1}$ is the $M \times 1$ vector of ones, $\mathbf{C}$ is the $M \times R \mathrm{SH}$ basis matrix whose $i$-th row is $\mathbf{C}_{i}=\left[Y_{1}\left(\vartheta_{i}, \varphi_{i}\right) \ldots Y_{R}\left(\vartheta_{i}, \varphi_{i}\right)\right], \mathbf{L}$ is the $R \times R$ diagonal matrix of Laplace-Beltrami eigenvalues with $\mathbf{L}_{j j}=-l_{j}\left(l_{j}+1\right)$, and $\mathbf{P}$ is the $R \times R$ diagonal Funk-Radon transform matrix with $\mathbf{P}_{j j}=2 \pi P_{l_{j}}(0)$. Thus, to enforce the non-negativity of $\boldsymbol{p},[10$ ] solves the optimization problem $\min _{\boldsymbol{c}}\|\mathbf{B} \boldsymbol{c}-\boldsymbol{s}\|_{2}^{2}$ subject to $\mathbf{C L P} \boldsymbol{c} \geq-4 \pi \mathbf{1}$. This method enforces $p_{L}\left(\vartheta_{i}, \varphi_{i}\right) \geq 0$ for $i=1, \ldots, M$ but not for all $\vartheta \in[0, \pi], \varphi \in[0,2 \pi)$.

\section{Estimating ODFs with Non-negativity Constraints}

In this section, we propose an algorithm for solving Problem 1, In \$3.1 we show that $b \doteq \min _{\vartheta, \varphi} p_{L}(\vartheta, \varphi)=\lim _{\ell \rightarrow \infty} \lambda_{1}^{\ell}$, where $\lambda_{1}^{\ell}$ is the smallest eigenvalue of

\footnotetext{
${ }^{1}$ Typically we consider $M=162$ with $G \approx 100$ HARDI measurements.
} 
a matrix $T_{\ell}$ constructed from the $\mathrm{SH}$ coefficients, $\overline{\tilde{\boldsymbol{c}}}$, of $p_{L}$. Therefore, enforcing $b \geq 0$ is equivalent to enforcing $\lambda_{1}^{\ell} \geq 0$ for all $\ell \geq L$. Unfortunately, we cannot solve an optimization problem with infinitely many constraints. Also, enforcing $\lambda_{1}^{\ell} \geq 0$ for a finite $\ell \geq L$ is a necessary but not a sufficient condition. To circumvent this problem, in $\$ 3.2$ we show that the sequence $\left\{\lambda_{1}^{\ell}\right\}_{\ell \geq L}$ is decreasing and that $b$ can be predicted by fitting a curve to the first few $\left\{\bar{\lambda}_{1}^{\ell}\right\}_{\ell=L}^{N}$. In $\$ 3.3$, we show that given $b$, one can estimate $\overline{\boldsymbol{c}}$ by solving an SDP. We thus propose an iterative SDP that alternates between computing $\overline{\boldsymbol{c}}$ given $b$ and predicting $b$ given $\overline{\tilde{c}}$.

\subsection{Toeplitz Form Analogue for Spherical Harmonic Basis}

In [12], results are developed which relate the range of the values of a function to the eigenvalues of a Toeplitz matrix (or form) constructed from finitely many Fourier coefficients of the function. One can develop analogue results for the SH coefficients. We follow [13] in constructing a matrix $T_{\ell}$ that serves as the $\mathrm{SH}$ analogue of the Toeplitz form in the Fourier case.

Consider a spherical function $f=\sum_{j=1}^{\infty} c_{i} Y_{i}$ and let $\overline{\boldsymbol{c}}=\left[c_{l, m}\right]$ be its standard SH coefficients of infinite length. Let $T_{\ell}(f), \ell=0,1, \ldots$, be a matrix whose rows and columns are indexed by the pair $\left(l_{1} m_{1}, l_{2} m_{2}\right)=\left(l_{1}\left(l_{1}+1\right)+m_{1}, l_{2}\left(l_{2}+1\right)+\right.$ $\left.m_{2}\right)$, where $l_{i}=0,1,2, \ldots, \ell$ and $-l_{i} \leq m_{i} \leq l_{i}$, for $i=1,2$. The entry of $T_{\ell}$ at position $\left(l_{1} m_{1}, l_{2} m_{2}\right)$ is defined as

$$
T_{\ell}(f)_{l_{1} m_{1} ; l_{2} m_{2}}=\sum_{l=\left|l_{1}-l_{2}\right|}^{l_{1}+l_{2}} \bar{c}_{l, m_{1}-m_{2}} G\left(l, l_{2}, l_{1} ; m_{1}-m_{2}, m_{2}, m_{1}\right),
$$

where $G\left(l_{1}, l_{2}, l_{3} ; m_{1}, m_{2}, m_{3}\right)$ is a real constant Gaunt Coefficient (See [13] Appendix A). The size of $T_{\ell}$ is $(\ell+1)^{2} \times(\ell+1)^{2}$ because there are $(\ell+1)^{2} \mathrm{SH}$ coefficients of degree less than or equal to $\ell$. The following result proved in [13] relates the extremal eigenvalues of $T_{\ell}$ to the range of the values of the function $f$ and in particular the behavior as $\ell \rightarrow \infty$.

Theorem 1. (Eigenvalue Distribution Theorem in $\left.\mathbb{S}^{2}\right)$ Let $f(u) \in L^{1}\left(\mathbb{S}^{2}\right)$ be an absolutely integrable real valued function on the 2-sphere. Let $b$ and $B$ be the essential lower and upper bounds of $f(u)$, respectively and let $\lambda_{i}^{\ell}, i=1, \ldots,(\ell+$ $1)^{2}$, be the ascending eigenvalues of the matrix $T_{\ell}(f)$. Then we have

$$
b \leq \lambda_{1}^{\ell} \leq \ldots \leq \lambda_{(\ell+1)^{2}}^{\ell} \leq B, \quad \lim _{\ell \rightarrow \infty} \lambda_{1}^{\ell}=b, \quad \text { and } \quad \lim _{\ell \rightarrow \infty} \lambda_{(\ell+1)^{2}}^{\ell}=B .
$$

This theorem shows that the smallest eigenvalue of $T_{\ell}(f)$ in the limit converges to the minimum of $f$ on $\mathbb{S}^{2}$. Therefore, enforcing that $\min _{\vartheta, \varphi} f(\vartheta, \varphi) \geq 0$, is equivalent to enforcing that $\lambda_{1}^{\ell} \geq 0$ for all $\ell \geq 0$.

\subsection{Predicting the Smallest Eigenvalue}

Since we cannot enforce infinitely many constraints, let us first understand the behavior of the smallest eigenvalue of $T_{\ell}\left(f_{L}\right)$ for $\ell \geq L$, where $f_{L}$ belongs to the class of functions expressed by $\mathrm{SH}$ of degree up to $L$, i.e., $f_{L}=\sum_{j=1}^{R} c_{j} Y_{j}$. 
Theorem 2. Let $f_{L}=\sum_{i=1}^{R} c_{i} Y_{i}$, where $R=\frac{(L+1)(L+2)}{2}$. Let $T_{\ell}\left(f_{L}\right)$ be defined as in (4) and let $\lambda_{1}^{\ell}$ be its smallest eigenvalue. Then $\lambda_{1}^{\ell+1} \leq \lambda_{1}^{\ell}$ for all $\ell \geq L$.

Proof. By [13], $T_{\ell}\left(f_{L}\right)$ is Hermitian for every $\ell \geq 0$. Then, by interlacing [14, p. 189], $\lambda_{1}^{\ell}$ will be less than or equal to the minimum eigenvalue of every principal submatrix of $T_{\ell}\left(f_{L}\right)$. Thus it suffices to show that $T_{\ell}\left(f_{L}\right)$ is a principal submatrix of $T_{\ell+1}\left(f_{L}\right)$ for all $\ell \geq L$. $T_{\ell}\left(f_{L}\right)$ is of size $(\ell+1)^{2} \times(\ell+1)^{2}$ and $T_{\ell+1}\left(f_{L}\right)$ is of size $(\ell+2)^{2} \times(\ell+2)^{2}$. If we restrict our matrix $T_{\ell+1}\left(f_{L}\right)$ to the first $(\ell+1)^{2}$ columns and rows, we denote this principal submatrix by $P_{\ell+1}\left(f_{L}\right)$. In (4) for $T_{\ell+1}\left(f_{L}\right), l_{1}$ and $l_{2}$ each range from 0 to $\ell+1$ and so the upper limit $l_{1}+l_{2}$ of the summation over $l$ will range from 0 to $2 \ell+2$. But from the zero-padding construction of $T_{\ell+1}\left(f_{L}\right)$ we see that coefficients after $2 \ell$ equal 0 . Thus this summation reduces to the range 0 to $2 \ell$ which is exactly equal to $T_{\ell}\left(f_{L}\right)$. So $T_{\ell}\left(f_{L}\right)$ is a principal submatrix of $T_{\ell+1}\left(f_{L}\right)$ for all $\ell \geq L$.

This result guarantees monotone decrease of $\lambda_{1}^{\ell}$. However, to predict $\lim _{\ell \rightarrow \infty} \lambda_{1}^{\ell}$ we need the rate of convergence, which is not straightforward to analyze. In the $1 \mathrm{D}$ Fourier case, one can argue that this rate is roughly proportional to $\frac{1}{\ell+2}[12$, pp. 65-67 and p. 72]. However, there are technical difficulties in carrying this argument over to $\mathbb{S}^{2}$. Nevertheless, in the 2D Fourier case one could argue that the rate is proportional to $\frac{1}{(\ell+2)^{2}}$. We use this intuition [12, 13] to obtain a good estimate of the rate of convergence. Given a sequence of eigenvalues, $\lambda_{1}^{\ell}$, we fit functions of the form $q \frac{1}{(\ell+d)^{r}}+b$, with free parameters $q, r, b$ for different values of $d$ and small values of $\ell>L$. In addition we compare them to an exponential curve of the form $q e^{-r \ell}+b$. Table 1 shows the $R^{2}$ goodness of fit value and exponent $r$ for different values of $d$ averaged over 100 samples of synthetic ODFs for $\ell=4, \ldots, 15$. We found that $d \approx 2$ and $1 \leq r \leq 2$ yield a satisfactory fit and support our intuition. In our experiments we chose $d=2$ and let $r$ be free.

\subsection{Iterative Semi-definite Programming Optimization Algorithm}

In this section, we formulate Problem 1 in terms of constraints on $T_{\ell}\left(p_{L}\right)$. Ideally, the optimization problem we want to solve is:

$$
\min _{\boldsymbol{c}}\|\mathbf{B} \boldsymbol{c}-\boldsymbol{s}\|_{2}^{2} \text { s.t. } T_{\ell}\left(p_{L}\right) \succeq 0, \quad \forall \ell \geq L,
$$

where $\boldsymbol{c} \in \mathbb{R}^{R}$. By Theorem 1 constraining $T_{\ell}\left(p_{L}\right) \succeq 0$ for all $\ell \geq L$ enforces $p_{L}(\vartheta, \varphi)=\sum_{j=1}^{R} \tilde{c}_{j} Y_{j}(\vartheta, \varphi) \geq 0$ for all $\vartheta \in[0, \pi], \varphi \in[0,2 \pi)$. It is important to note here that since $p_{L}$ has $\mathrm{SH}$ coefficients $\tilde{\boldsymbol{c}}, T_{\ell}\left(p_{L}\right)$ will be built from $\overline{\tilde{\boldsymbol{c}}}=\left[\tilde{c}_{l, m}\right]$.

Table 1. Rate of Convergence Parameters and Performance

\begin{tabular}{|c|ccccc|c|}
\hline$d$ & 0 & 1 & 2 & 2.5 & 3 & $q e^{-r \ell}+b$ \\
\hline$R^{2}$ & 0.9989 & 0.9990 & 0.9991 & 0.9991 & 0.9991 & 0.4522 \\
\hline$r$ & 0.7678 & 1.0162 & 1.2606 & 1.3819 & 1.5026 & 4.0142 \\
\hline
\end{tabular}




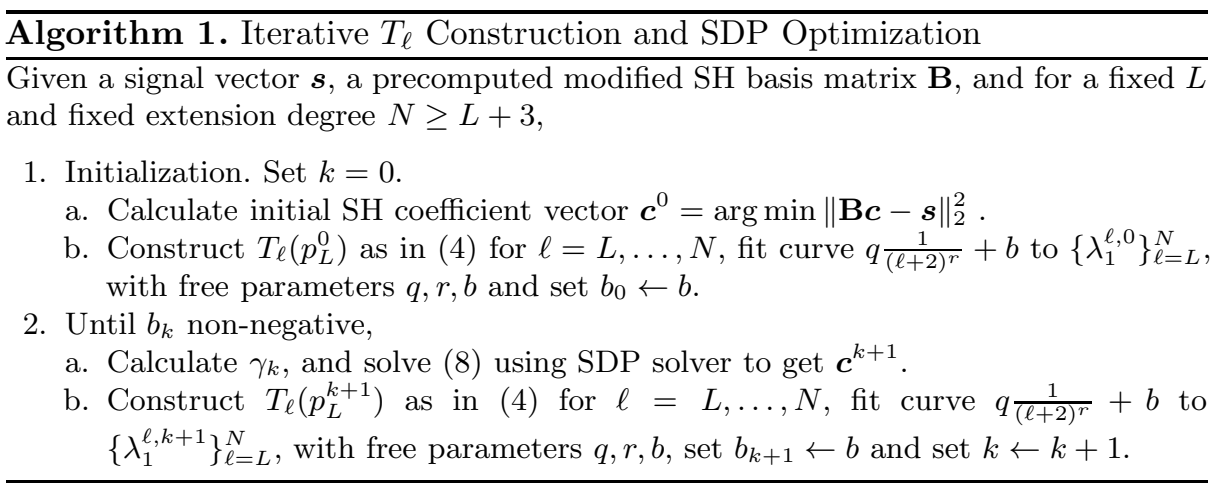

Notice that we cannot solve the problem in (6), because it has infinitely many constraints. Notice also that enforcing $T_{\ell}\left(p_{L}\right) \succeq 0$ for finite $\ell$ does not guarantee the non-negativity of $p_{L}$. To address this issue, we propose an iterative algorithm, (see Alg. 11) that solves an approximation of (6) at each iteration. Let $c^{k}$ be the estimate of $\boldsymbol{c}$ at iteration $k$ and define $p_{L}^{k}=\sum_{j=1}^{R} \tilde{c}_{j}^{k} Y_{j}$. Let $\lambda_{1}^{\ell, k}$ be the minimum eigenvalue of $T_{\ell}\left(p_{L}^{k}\right)$ and let $b^{k}$ be the prediction of $\lim _{\ell \rightarrow \infty}\left\{\lambda_{1}^{\ell, k}\right\}$ obtained by the curve fitting method described in $\$ 3.2$. At iteration $k$, we solve:

$$
\boldsymbol{c}^{k+1}=\underset{\boldsymbol{c}}{\arg \min }\|\mathbf{B} \boldsymbol{c}-\boldsymbol{s}\|_{2}^{2} \text { s.t. } T_{L}\left(p_{L}^{k}\right) \succeq \gamma_{k} \mathbf{I}_{(L+1)^{2}},
$$

where $\gamma_{k} \doteq \lambda_{1}^{L, k}-b_{k}$ and $\mathbf{I}_{(L+1)^{2}}$ is the identity matrix of size $(L+1)^{2}$. Alg. 11 was implemented in MATLAB utilizing the Coder Toolbox to speed up construction of $T_{\ell}$. The optimization problem was solved using CVX, a MATLAB software for specifying and solving convex programs [15, 16]. Within the CVX environment (77) is reformulated using the Shur complement of the constraint $\|\mathbf{B} \boldsymbol{c}-\boldsymbol{s}\|_{2}^{2} \leq z$ as:

$$
\min _{\boldsymbol{c}} z \text { s.t. } \quad\left[\begin{array}{cc}
\mathbf{I}_{R} & \mathbf{B} \boldsymbol{c}-\boldsymbol{s} \\
{[\mathbf{B} \boldsymbol{c}-\boldsymbol{s}]^{T}} & z
\end{array}\right] \succeq 0 \quad \text { and } \quad T_{L}\left(p_{L}^{k}\right) \succeq \gamma_{k} \mathbf{I}_{(L+1)^{2}}
$$

for $z \in \mathbb{R}$. In Alg. 1, we begin with a given measured signal $s$ and modified $\mathrm{SH}$ basis matrix $\mathbf{B}$ as described in $\$ 2.2$. To initialize the algorithm, we fix $L$ and start with a least squares approximation for $c$ as in step 1a, From the initial optimal value $\boldsymbol{c}^{0}$ we can calculate $\overline{\tilde{\boldsymbol{c}}}^{0}$, by the mapping in $\$ 2$, in order to construct $T_{L}\left(p_{L}^{0}\right)$ by (4). We can optimize over any $T_{\ell}$ so we choose the smallest $\ell=L$ for computational simplicity. For a fixed integer $N \geq L+3$ we fit the curve to $\left\{\lambda_{1}^{\ell, 0}\right\}$ for $\ell=L, \ldots, N$ as in $\$ 3.2$ in order to calculate the approximate minimum $b=b_{0}$ of the function $p_{L}^{0}$. If $b_{0}<0$ we then solve the problem in (8), which will increase the expected minimum of the sequence $\left\{\lambda_{1}^{\ell, k+1}\right\}$, generated from the optimal coefficients of the optimization, closer to 0 . Alg. 1 1 repeats until we arrive at a $b_{k} \geq 0$, which means we have a function $p_{L}^{k} \geq 0$ on the continuous domain. 


\section{Experiments}

We conducted a number of experiments on real and synthetic datasets to compare our continuous non-negativity $(\mathrm{CN})$ method against the discrete non-negativity (DN) method [10] and the unconstrained least squares (LS) method. Our algorithm uses (DN) as initialization. We used the multi-tensor method in [4] to generate a field of 100 synthetic ODFs with 1,2 , and 3 fibers with varying angles. We added complex Gaussian noise with a signal-to-noise ratio (SNR) of 5, 10 and $20 \mathrm{~dB}$. Alg. 1 takes on avg. 2-3 iterations to converge. Each iteration in MATLAB takes about 70 s for $N=15$ and 159 s for $N=20$, thus we choose $N=15$ in our experiments. This is about two orders of magnitude more than the method of [10], which takes 1s per ODF. The runtime of our method could be reduced by using a more efficient implementation than CVX. The computational complexity of our SDP is almost $O\left(L^{6}\right)$. Fig. 1 1 offers a closeup investigation of a synthetic single fiber ODF with SNR $5 \mathrm{~dB}$, using each method. Notice that CN more closely estimates the true ODF. To quantify the error, we used the Riemannian distance between ODFs, dist ${ }_{\text {Rie }}$ [17. All experiments are calculated over a very fine mesh of 1.002 million points on $\mathbb{S}^{2}$. The left plot in Fig. 2 shows the avg. Riemannian error between the true and estimated ODFs for each SNR value. The avg. error of $\mathrm{CN}$ is consistently lower than that of DN. Further analysis in the righthand plot in Fig. 2 presents the avg. percentage of negative values on $\mathbb{S}^{2}$ for each SNR level for LS, DN, and DN with two passes of spatial regularization (DN-S) [10]. The number of negative values decreases but is not eliminated, while in CN $0 \%$ of the ODFs have negative values over the fine mesh, and in theory for all points on $\mathbb{S}^{2}$. Finally, we validate our methods on a $128 \times 128$ real HARDI human brain dMRI dataset measured at 94 gradient directions. Fig. 3 compares DN and CN on a sample of the real dataset. Using the same mesh as in the synthetic experi-

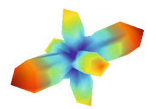

(a) LS

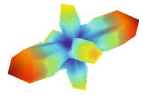

(b) DN

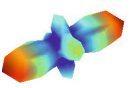

(c) $\mathrm{CN}$

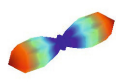

(d) True

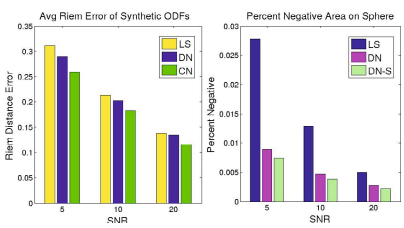

Fig. 1. Single fiber ODF with SNR $5 \mathrm{~dB}$
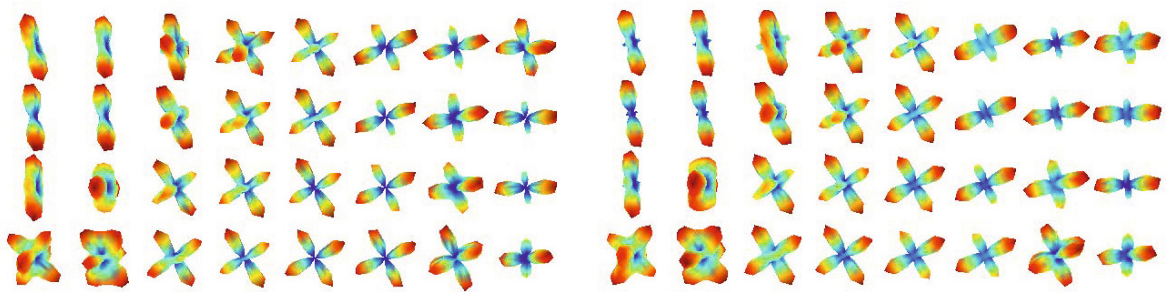

Fig. 3. Real HARDI brain ODF field. Left: DN, Right: CN 
ments, we found that over $99 \%$ of the ODFs estimated by DN had on avg. $0.04 \%$ of negative values, while $0 \%$ of the ODFs estimated by $\mathrm{CN}$ had negative values.

\section{Conclusion}

We have proposed a novel method to enforce non-negativity in the estimation of ODFs. We not only eliminate the negative values of existing estimation methods, but also improve the estimated ODFs, as demonstrated with synthetic and real experiments. Future work includes using our axiomatically correct ODFs to demonstrate improvement in statistical analysis for population studies.

Acknowledgments. This work was supported in part by JHU startup funds.

\section{References}

1. Frank, L.R.: Characterization of anisotropy in high angular resolution diffusionweighted MRI. Magnetic Resonance in Medicine 47(6), 1083-1099 (2002)

2. Özarslan, E., Mareci, T.: Generalized diffusion tensor imaging and analytical relationships between diffusion tensor imaging and high angular resolution diffusion imaging. Magnetic Resonance in Medicine 50, 955-965 (2003)

3. Hess, C.P., Mukherjee, P., Han, E.T., Xu, D., Vigneron, D.B.: Q-ball reconstruction of multimodal fiber orientations using the spherical harmonic basis. Magnetic Resonance in Medicine 56(1), 104-117 (2006)

4. Descoteaux, M., Angelino, E., Fitzgibbons, S., Deriche, R.: Regularized, fast and robust analytical Q-ball imaging. Mag. Res. in Med. 58(3), 497-510 (2007)

5. Jian, B., Vemuri, B.: A unified computational framework for deconvolution to reconstruct multiple fibers from diffusion weighted MRI. IEEE Transactions on Medical Imaging 26(11), 1464-1471 (2007)

6. Tristan-Vega, A., Westin, C.F., Aja-Fernandez, S.: Estimation of fiber orientation probability density functions in high angular resolution diffusion imaging. NeuroImage 47(2), 638-650 (2009)

7. Aganj, I., Lenglet, C., Sapiro, G., Yacoub, E., Ugurbil, K., Harel, N.: Reconstruction of the orientation distribution function in single- and multiple-shell q-ball imaging within constant solid angle. Magnetic Resonance in Medicine 64(2), 554$566(2010)$

8. Qi, L., Yu, G., Wu, E.: Higher order positive semidefinite diffusion tensor imaging. SIAM J. Imaging Sci. 3 (2010)

9. Barmpoutis, A., Vemuri, B.: A unified framework for estimating diffusion tensors of any order with symmetric positive-definite constraints. In: IEEE International Symposium on Biomedical Imaging, pp. 1385-1388 (2010)

10. Goh, A., Lenglet, C., Thompson, P., Vidal, R.: Estimating Orientation Distribution Functions with Probability Density Constraints and Spatial Regularity. In: Yang, G.-Z., Hawkes, D., Rueckert, D., Noble, A., Taylor, C. (eds.) MICCAI 2009, Part I. LNCS, vol. 5761, pp. 877-885. Springer, Heidelberg (2009)

11. Tournier, J.D., Calamante, F., Connelly, A.: Robust determination of the fibre orientation distribution in diffusion MRI: Non-negativity constrained super-resolved spherical deconvolution. NeuroImage 35(4), 1459-1472 (2007) 
12. Grenander, U., Szego, G.: Toeplitz Forms and their Applications. University of California Press (1958)

13. Shirdhonkar, S., Jacobs, D.: Non-negative lighting and specular object recognition. In: IEEE Conference on Computer Vision and Pattern Recognition (2005)

14. Horn, R.A., Johnson, C.R.: Matrix Analysis. Cambridge University Press (1985)

15. Grant, M., Boyd, S.: CVX: Matlab software for disciplined convex programming, version 1.21 (April, 2011), http://cvxr.com/cvx/

16. Grant, M., Boyd, S.: Graph implementations for nonsmooth convex programs. In: Blondel, V., Boyd, S., Kimura, H. (eds.) Recent Advances in Learning and Control. LNCIS, vol. 371, pp. 95-110. Springer, Heidelberg (2008)

17. Goh, A., Lenglet, C., Thompson, P., Vidal, R.: A nonparametric Riemannian framework for processing high angular resolution diffusion images and its applications to ODF-based morphometry. NeuroImage 56(1), 1181-1201 (2011) 